\title{
Doppler echocardiography in elderly patients with ejection systolic murmurs
}

\author{
G.M. McKillop' ${ }^{1}$ D.A. Stewart ${ }^{2}$, J.M.A. Burns ${ }^{2}$ and D. Ballantyne ${ }^{1}$ \\ ${ }^{1}$ Department of Medicine, Victoria Infirmary, Glasgow and ${ }^{2}$ Victoria Geriatric Unit, Glasgow, UK
}

\begin{abstract}
Summary: Thirty-nine elderly patients, mean age 77 years (range 65 to 96), with ejection systolic murmurs were studied to evaluate the functional significance of these murmurs. Subjects were evaluated clinically, by 2-D echocardiography, and by a full Doppler echocardiography study. Good quality Doppler signals were obtained in 35 subjects. Mitral regurgitation was found to be the only significant valvular lesion in 6 patients (17\%). Doppler gradients in systole across the aortic valve were less than $30 \mathrm{mmHg}$ in 28 subjects $(80 \%)$ and were considered not significant. Gradients of greater than $30 \mathrm{mmHg}$ representing significant aortic stenosis were found in 7 subjects (20\%). The clinical sensitivity in detecting significant aortic stenosis was $44 \%$ and specificity was $81 \%$. Doppler evidence of significant aortic stenosis was found in a substantial proportion of these elderly subjects. Neither clinical assessment nor 2-D echocardiography can be relied on to exclude this condition.
\end{abstract}

\section{Introduction}

Aortic systolic murmurs are found in approximately $50 \%$ of elderly patients. ${ }^{1,2}$ Establishing the aetiology of these murmurs and differentiating between significant aortic stenosis and the debated diagnosis of aortic sclerosis is difficult on clinical and electrocardiographic grounds alone. The introduction of echocardiography, both M-mode and real time, helped to evaluate this problem but relies on indirect measures including left ventricular internal diameter, wall thickness and aortic valve calcification. Measurements of valvular gradients and their precise anatomical localization, hitherto only possible with invasive procedures, are now possible to assess using Doppler echocardiography.

The aims of this study were: to determine the significance of ejection systolic murmurs in the elderly; to appraise the sensitivity and specificity of clinical assessment in detecting significant disease; and to relate clinical and electrocardiographic findings to aortic valve function as gauged by Doppler echocardiography.

\section{Methods}

Thirty-nine elderly subjects, mean age 77 years (range 65 to 96 years), were studied. Recruitment

\footnotetext{
Correspondence: G.M. McKillop, M.R.C.P., Department of Radiology, Victoria Infirmary, Langside, Glasgow G42 9TY, UK.

Accepted: 8 February 1991
}

was from among both general medical and geriatric inpatients and outpatients and from attenders at a geriatric day hospital. Consecutive subjects with ejection systolic murmurs of any grade as judged by attending medical staff were recruited regardless of cardiac symptoms. Patients with overt cardiac failure were excluded as were those unable to cooperate with echocardiography.

Patients were assessed by a cardiologist and by a geriatrician. Grade of murmur, quality of the aortic second sound (reduced, normal, or increased), the presence or absence of aortic regurgitation, and finally a verdict on the presence or absence of significant aortic stenosis were recorded independently by the two clinicians, along with blood pressure and pulse pressure.

A 12 lead electrocardiogram (ECG) was recorded and analysed for voltage criteria of left ventricular hypertrophy, ${ }^{3}$ electrical axis and evidence of conduction defects. 2-D and Doppler (continuous wave) echocardiography were recorded using a Hewlett Packhard phased array $90^{\circ}$ sector scanner $(3.5 \mathrm{MHz}$ transducer). Echocardiograms were recorded on VHS videotape and analysed later for valvular abnormalities (aortic stenosis or aortic regurgitation), aortic valve calcification and cusp separation on 2-D and aortic valve gradient in systole and the presence of aortic regurgitation on Doppler. All echocardiographic and Doppler data was averaged over 3 cardiac cycles.

Adequate quality echocardiograms were obtained in 35 subjects with full clinical and ECG data 
in 31. Doppler gradients across the aortic valve in excess of $30 \mathrm{mmHg}$ were regarded as representing significant stenosis and those under $20 \mathrm{mmHg}$ as not significant. Sensitivity and specificity of clinical detection of aortic stenosis by the two clinicians was evaluated.

Analysis of ECG and Doppler echocardiography data was performed using correlation coefficients and Wilcoxon's rank sum test.

\section{Results}

Of the 35 subjects with full echocardiographic data, 6 were found to have mitral regurgitation as the only significant valvular pathology. Of the 29 remaining, 22 had gradients of less than $20 \mathrm{mmHg}$ and 7 had gradients in excess of $30 \mathrm{mmHg}$ with no subject between these values (Table I).

The clinical sensitivity in detecting significant stenosis was $44 \%$ and the specificity was $81 \%$. There was no significant association between the presence of stenosis as defined by Doppler gradients and either the grade of the murmur or the quality of the aortic second sound. Median pulse pressure was significantly lower in those with stenosis at $45 \mathrm{mmHg}$ (range 15-80) than those without at $65 \mathrm{mmHg}$. (range $35-100)(P<0.05)$.

There was a weak correlation between voltage measurements from ECG and the Doppler gradient $(r=0.4, P<0.05)$. ECG criteria of left ventricular hypertrophy, however, were of little value in predicting the presence of significant stenosis with a sensitivity of only $37.5 \%$. Neither cusp separation or the presence of valve calcification on 2-D echocardiography showed significant correlation with Doppler gradients.

\section{Discussion}

Real time Doppler echocardiography is a noninvasive method of evaluating valvular abnormalities and is now available in most teaching and in many district general hospitals. There is good correlation between aortic valve gradients measured at cardiac catheterization and those measured simultaneously by Doppler echocardiography. ${ }^{4,5}$ It was therefore felt justifiable to use the measured Doppler gradient as a 'gold standard' in this non-invasive study for evaluating ejection systolic murmurs in the elderly. Recourse to cardiac catheterization would be hard to justify on ethical grounds.

The gradient across the aortic valve which should be considered evidence of 'significant' stenosis is difficult to define and no universally agreed level exists. Even with left heart catheterization, where gradients may be peak-to-peak or
Table I Doppler gradients (excluding 6 subjects with mitral regurgitation)

\begin{tabular}{|c|c|c|c|}
\hline & Number & $\begin{array}{c}\text { Range } \\
(\mathrm{mmHg})\end{array}$ & Median \\
\hline Total & 29 & $4-164$ & 10 \\
\hline $\begin{array}{l}\text { Non-significant } \\
\text { (gradient }<20 \mathrm{mmHg} \text { ) }\end{array}$ & 22 & $4-18$ & 8 \\
\hline $\begin{array}{l}\text { Significant } \\
\quad \text { (gradient }>30 \mathrm{mmHg} \text { ) }\end{array}$ & 7 & $38-164$ & 56 \\
\hline
\end{tabular}

instantaneous, there is no universally agreed gradient which represents significant stenosis. It is known that the gradient at rest depends on both left ventricular function and the level of patient anxiety, and may underestimate values during exercise. There is an intermediate level of velocity/gradient between normal flow and definite stenotic/high velocity flow. For the purposes of this study, $20 \mathrm{mmHg}$ was selected as representing stenosis. In practice, all 7 subjects considered to have stenosis had gradients in excess of $30 \mathrm{mmHg}$ and this would be accepted by most authorities as indicating significant disease. Maximum Doppler gradients ranging from $31 \mathrm{mmHg}$ to $111 \mathrm{mmHg}$ have beer reported in subjects with critical stenosis (aortie valve area $\left.<=0.75 \mathrm{~cm}^{2}\right){ }^{6}$

The significance of a finding of aortic stenosis in these patients is not clear. We believe we are the first to report on the prevalence of aortic stenosis in unselected patients with aortic murmurs, most of whom were not suspected clinically of having significant disease. Prior to the introduction of Doppler echocardiography there was no method of non-invasively screening for or accurately assessing the severity of aortic stenosis. Accordingly investigation has been limited to those in whom aortic stenosis was suspected clinically and who would be candidates for surgical intervention. Currently available information on disease progression and the need for intervention is based on experience with this highly selected group of patients and may not be applicable to the type of patients identified in this study. The prognosis and need for follow up of these patients requires further study.

This study confirms the unreliability of clinical assessment of aortic systolic murmurs in elderly patients. ${ }^{7}$ Further evidence of this is the fact that $17 \%$ of subjects considered to have ejection systolic murmurs had mitral regurgitation as the only significant finding. No significant associations between clinical or ECG parameters and the presence of stenosis were found indicating that, even with the addition of ECG evidence, clinical evaluation is insufficiently accurate. Pulse pressure was the only measurement showing a significant

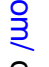
(n)

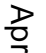
(2) (n)

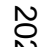

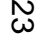
(2) 
difference between those with and without stenosis. There is a large degree of overlap between the two groups, however, and the usefulness of this as a predictor of stenosis is limited. 2-D echocardiography evidence (cusp separation and presence of valve calcification) also showed no correlation with significant stenosis indicating that the indirect measurements of valvular dysfunction provided by this technique are not reliable.

One of the problems with Doppler assessment of aortic valve gradient is that the actual measurement made is of velocity of blood flow which is converted into a gradient. This is the standard technique on all Doppler equipment and in practice involves marking the peak velocity on the display screen electronically. An automatic analysis is then performed using the Bernoulli equation to derive the gradient. ${ }^{8}$ When cardiac output is low, the ejection fraction is lower resulting in a decreased velocity of flow over the valve. This may result in the gradient across the valve being underestimated. In this study this was not found to be a problem as no subject had a fractional shortening of less than $25 \%$.

In summary, this study shows a high prevalence of significant aortic valve disease in elderly subjects with ejection systolic murmurs. The evaluation of these patients is difficult. A substantial number of patients have, in fact, mitral valve disease. In those with aortic murmurs neither clinical assessment, ECG evidence or 2-D echocardiography are sufficiently accurate to be useful. Reliable information on the presence or absence of significant aortic stenosis in these patients can be obtained non-invasively only by Doppler echocardiography.

\section{References}

1. Bruns, D.L. \& Van der Hauwert, L.G. The aortic systolic murmur developing with increasing age. Br Heart J 1958, 20: $370-378$.

2. Bethel, C.S. \& Crow, E.W. Heart sounds in the aged. Am J Cardiol 1963, 11: 763-767.

3. Rhomilt, D.W. \& Estes, D.H. A point score system for the ECG diagnosis of left ventricular hypertrophy. Am Heart $J$ 1968, 75: 752-758.

4. Yeager, M., Yock, P.G. \& Popp, R.L. Comparison of Doppler derived pressure gradient to that derived at cardiac catheterisation in adults with aortic valve stenosis: implications for management. Am J Cardiol 1986, 57: 644-648.

5. Smith, M.D., Kwan, O.L. \& Demaria, A.N. Value and limitations of continuous wave Doppler echocardiography in estimating severity of valvular stenosis. JAMA 1986, 255: $3145-3151$.

6. Danielsen, R., Nordrehaug, J.E., Stangeland, L. \& Vik-Mo, H. Limitations in assessing the severity of aortic stenosis by Doppler gradients. Br Heart J 1988, 59: 551-555.

7. Jaffe, W.D., Roche, J.H.D., Coverdale, H.A., McAllister, H.F., Ormiston, J.A. \& Greene, E.R. Clinical evaluation versus Doppler echocardiography in the quantitative assessment of valvular heart disease. Circulation 1988, 78: 267-275.

8. Hagler, D. Aortic valve disease. In: Houston, A.B. \& Simpson, I.A. (eds).Cardiac Doppler Ultrasound: A Clinical Perspective. Wright, London, 1988, pp. 43-48. 